\title{
Joint Interference and User Association Optimization in Cellular Wireless Networks
}

\author{
Changkyu Kim, Student Member, IEEE, Russell Ford, Student Member, IEEE, Yanjia Qi, Student Member, IEEE, \\ Sundeep Rangan, Senior Member, IEEE
}

\begin{abstract}
In cellular wireless networks, user association refers to the problem of assigning mobile users to base station cells - a critical, but challenging, problem in many emerging small cell and heterogeneous networks. This paper considers a general class of utility maximization problems for joint optimization of mobile user associations and bandwidth and power allocations. The formulation can incorporate a large class of network topologies, interference models, SNR-to-rate mappings and network constraints. In addition, the model can applied in carrier aggregation scenarios where mobiles can be served by multiple cells simultaneously. While the problem is nonconvex, our main contribution shows that the optimization admits a separable dual decomposition. This property enables fast computation of upper bounds on the utility as well as an efficient, distributed implementation for approximate local optimization via augmented Lagrangian techniques. Simulations are presented in heterogeneous networks with mixtures of macro and picocells. We demonstrate significant value of the proposed methods in scenarios with variable backhaul capacity in the femtocell links and in cases where the user density is sufficiently low that lightlyused cells can reduce power.
\end{abstract}

Index Terms - cellular wireless systems, user association, dual decomposition, inter-cell interference coordination, carrier aggregation, 3GPP LTE.

\section{INTRODUCTION}

In cellular wireless networks, user association refers to the problem of assigning each mobile terminal a serving base station cell. In traditional cellular systems, user association is relatively straightforward in that mobiles can simply connect to the cell with the strongest received signal strength. This simple selection policy maximizes the SNR to each user and hence the rate per unit bandwidth.

However, in many emerging cellular network deployment models, user association requires consideration of other factors in addition to signal strength. For example, to scale capacity in a cost effective manner, traditional macrocellular networks are being increasingly supplemented with overlays of smaller micro- and picocells [1], [2]. The resulting heterogeneous networks (HetNets) will consist of cells with vastly different sizes and require load balancing to encourage mobiles to connect to smaller cells that would otherwise be underutilized [3], [4]. In addition, these HetNets may also contain open femtocells [5], [6] where the wired connectivity would

This material is based upon work supported by the National Science Foundation under Grants No. 1116589 and 1237821.

C. Kim (email:ckim02@students.poly.edu), R. Ford (email:rford02@students.poly.edu), Y. Qi (email: yqi@poly.edu) S. Ranagn (email: srangan@poly.edu) are with the NYU Wireless Center, Polytechnic Institute of New York University, Brooklyn, NY. be provided by third parties other than the cellular provider. Third party backhaul capacity may be highly variable and may also need to be considered in the user association decisions. Finally, the state-of-the-art cellular standards offer a number of advanced intercellular interference coordination (ICIC) mechanisms including subband scheduling and beamforming [7]. As a result, the throughput a user can experience in any one cell can depend in a complex manner on the particular choice of user assignments and resource allocation decisions in other cells.

To understand these issues, this paper considers a general class of problems for joint optimization of user association and bandwidth and power allocations for interference coordination in cellular wireless networks. The joint user associationinterference problem is formulated in the classical framework of utility maximization [8], [9]. Specifically, the throughput to the users is regarded as a function of the cells that they are assigned to as well as the resources (power or bandwidth) allocated to the users in the cells. The bandwidth and power allocations in one cell may affect the interference levels in other cells. The problem is to match the users to cells and allocate resources within each cell to control the inter-cellular interference and maximize some system-wide utility function of the rates.

Two variants of the problem are considered: (a) Multiflow optimization where mobiles can be served by multiple cells simultaneously as in the case of 3GPP LTE with carrier aggregation (CA) [10], [11]; and (b) user association optimization where each mobile can only be served by one base station cell at a time as in the case of standard 3GPP LTE without advanced CA capability.

Both problems are, in general, non-convex. However, while we cannot find an optimal solution, our main contribution shows that under very general formulations, the multiflow optimization admits a separable dual decomposition. This separability property has several key implications: First, for any given values of the Lagrange parameters, the dual problem can be solved easily and hence one can minimize a dual upper bound exactly. Therefore, we can obtain a computable upper bound against which we can compare the performance of any practical, suboptimal algorithm. Secondly, although the problem may have a non-zero duality gap (i.e. the problem may not be strongly dual), the separability of the dual problem enables efficient implementation of various augmented Lagrangian techniques for constrained maximization. Finally, dual techniques have the benefits that they readily lead to distributed implementations where the base stations broadcast 
the dual parameters and the mobiles update the cell selections. In the multiflow problem, the Lagrange parameters have a natural interpretation as access prices.

For user association, we recognize that the problem is essentially classic route selection in networking theory. Route selection is known in general to be NP-hard, but we follow a standard procedure where we use the solution to the multiflow problem as an upper bound and then truncate the solution for the single path constraint $[12]-[14]$. In this way, we can apply dual decomposition plus one additional truncation step for the user association problem. This mutliflow approximation has also been used in cellular user association in [4] (see below).

An appealing feature of our approach is that the dual separability applies under very general circumstances. Specifically, our formulation incorporates a general linear mixing interference model, first presented in [15] for ICIC problems in femtocells. Although we will only study bandwidth and power allocations in this paper, the model can incorporate - with some extensions - other ICIC techniques including subband allocations and beamforming. In addition, our only restriction on the utility functions is that they are separable. The dual decomposition will hold even under non-concave utilities. Finally, unlike previous formulations, the approach here can incorporate network constraints (provided they are known) including limits in the backhaul - an important feature for third-party offload.

\section{A. Related Work}

Early work in the area of optimal user association includes the classic paper by Hanly [16] that found a simple iterative algorithm for jointly optimal user association and uplink power control in CDMA systems. Downlink versions of this problem were later considered in [17]. Although we will focus on bandwidth allocation for OFDMA systems in this paper, our methods apply to general linear mixing interference models, and thus power control may also be in considered in the future.

The recent interest in user association is however not for power control, but rather optimized load balancing in heterogeneous networks as described in [3], [4], [18]. Solutions in 3GPP standards bodies have focussed on simple "range expansion" techniques that apply a fixed bias in the cell selection procedure to shift mobiles to the smaller cells [19], [20]. These techniques can be supplemented with adaptive bias based on SINR [3] and iterative transmit power selection [18]. The cell-site selection problem is addressed as utility-based optimization problems with backhaul capacity constraints in [21], while [22] proposes a distributed algorithm based on an optimal load of base stations. Although our evaluation methodology is based on standard simulation models such as [23], stochastic analyses have been provided for these range expansion techniques in [24]. The methodology in this paper follows most closely to the work [4] that used a similar optimization technique with a multiflow upper bound and dual decomposition. The work [4] however does not consider joint optimization of user association with power and bandwidth control.

One of the interesting findings in [3], [4], [18] was that static range expansion works remarkably well and more sophisti- cated optimization approaches offer little benefit. However, here we will see that, when combined with joint bandwidth / power optimization, or in the presence of network constraints, optimized user association can offer larger gains in some scenarios. For example, as our simulations will indicate, when the network loading per cell is light or when the backhaul capacity is limited, unused cells can reduce power improving the interference conditions in other cells.

To perform the joint optimization of user association and interference coordination, our work synthesizes several wellknown network optimization methods. The use of multipath upper bounds for optimized route selection is a classic technique in networking theory that dates at least back to [12], [13] - see the text [14] for a historical survey as well as some testable conditions under which the multipath upper bound is provable optimal. See also [8], [25]-[27]. Joint power and congestion problems is given in the well-known paper [28]. See also [29] and the monograph [30]. This works also uses dual decomposition techniques, but applies to a potentially more general linear mixing interference model that includes power control as a special. However, while more general, our methodology has no guarantee of global optimality.

Utility optimization approaches to the multipath problems have been extensively studied including both primal and dual solutions with close connections to rate control [8] and network pricing [25]. The dual decomposition methods used in this paper are closest to [26], [27] which explore augmented Lagrangian and method of multiplier techniques.

\section{General Flow And Interference Control PROBLEM}

Before describing the user association and multiflow problems in cellular networks, we first describe a general joint flow control and interference optimization problem. We will apply this general formulation to cellular networks in the next section.

For the general problem, we consider a system with $S$ flows with rates $r_{s}, s=1, \ldots, S$ where a subset of the flows are constrained by interfering wireless links. Index the wireless links by $\ell=1, \ldots, L$ and let $\Gamma(\ell)$ denote the set of flows that share the wireless link $\ell$. We assume that wireless link capacity places a constraint on the rates through the link of the form

$$
\sum_{s \in \Gamma(\ell)} r_{s} \leq C_{\ell}\left(x_{\ell}, z_{\ell}\right), \quad \ell=1, \ldots, L,
$$

where $x_{\ell}$ denotes a resource allocation variable for the link, $z_{\ell}$ is the interference level on that link and $C_{\ell}\left(x_{\ell}, z_{\ell}\right)$ is the wireless link capacity. Following [15], our key assumption will be that the interference level on the links are a linear function of the resource allocation variables on the other links so that we can write

$$
\mathbf{z}=\mathbf{G} \mathbf{x},
$$

for some gain matrix G. In addition, we assume that there are some linear constraints on the resource allocation variables of the form

$$
\mathbf{A}_{x} \mathbf{x} \leq \mathbf{b}_{x}
$$




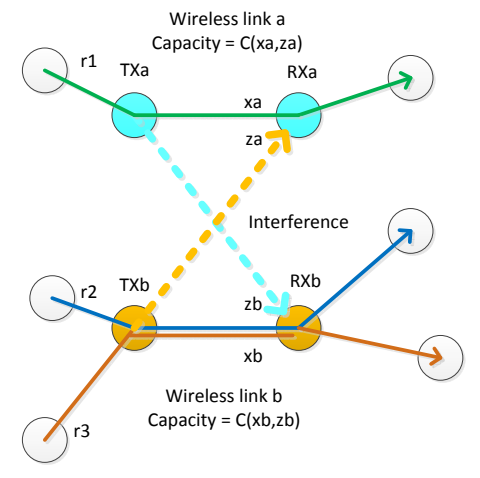

Fig. 1: Simple example of a network with three flows and two interfering wireless links for joint power control and rate optimization.

for some matrix $\mathbf{A}_{x}$ and vector $\mathbf{b}_{x}$. In the sequel, it will be convenient to rewrite the $L$ constraints (1) in a single matrix form

$$
\mathbf{E r} \leq C(\mathbf{x}, \mathbf{z})
$$

where $\mathbf{E}$ is the incidence matrix with components

$$
E_{\ell s}= \begin{cases}1 & \text { if } s \in \Gamma(\ell), \\ 0 & \text { if } s \notin \Gamma(\ell),\end{cases}
$$

and $C(\mathbf{x}, \mathbf{z})$ is the capacity vector

$$
C(\mathbf{x}, \mathbf{z})=\left(C_{1}\left(x_{1}, z_{1}\right), \cdots, C_{L}\left(x_{L}, z_{L}\right)\right)^{T} .
$$

In the user selection problem below, $x_{\ell}$ will be a quantity proportional to the power allocated to a wireless link $\ell$. Hence the interference powers $z_{\ell}$ are naturally described as a linear function of the form (2) where the matrix $\mathbf{G}$ would depend on the path losses between the transmitters and receivers. The link capacity (1) could then be a function of the signal-tonoise ratio $x_{\ell} / z_{\ell}$. As described in [15], the linear mixing interference model 22 can also be used for studying more complex interference scenarios including links with subbands or beamforming.

As discussed in the Introduction, we also wish to incorporate rate constraints in the wired network. To this end, we assume the network constraints are of the form [14]

$$
\mathbf{A}_{r} \mathbf{r} \leq \mathbf{b}_{r}
$$

for some matrix $\mathbf{A}_{r}$ and vector $\mathbf{b}_{r}$.

Our goal is to select a resource allocation vector $\mathbf{x}$ and flow rate vector $\mathbf{r}$ to maximize some utility function of the form

$$
U(\mathbf{r}):=\sum_{s=1}^{S} U_{s}\left(r_{s}\right),
$$

where $U_{s}\left(r_{s}\right)$ is the utility of the rate on flow $s$. Our formulation will not require that the utility functions are concave, only that they are continuous and monotonically increasing in $\mathbf{r}$.

To illustrate the formulation, Fig. 1 shows a simple example for joint power control and rate optimization. The network has $S=3$ flows with rates $r_{1}, r_{2}$ and $r_{3}$. Two of the links, labeled links $a$ and $b$, are wireless links that interfere with one another. The transmit signal powers on the links are denoted $x_{a}$ and $x_{b}$, while $z_{a}$ and $z_{b}$ represent the interference powers. In this case,

$$
z_{a}=G_{a b} x_{b}, \quad z_{b}=G_{b a} x_{a},
$$

where $G_{a b}$ and $G_{b a}$ are the cross gains between the transmitters and receivers. The SINRs on the two links would be given by

$$
\gamma_{a}=\frac{G_{a a} x_{a}}{z_{a}+n_{a}}, \quad \gamma_{b}=\frac{G_{b b} x_{b}}{z_{b}+n_{b}}
$$

where $n_{a}$ and $n_{b}$ are the noise powers and $G_{a a}$ and $G_{b b}$ are the gains on the intended links. If the link capacities $C_{a}$ and $C_{b}$ can be modeled as functions of the SINRs $\gamma_{a}$ and $\gamma_{b}$, then we can write

$$
r_{1} \leq C_{a}=C_{a}\left(x_{a}, z_{a}\right), \quad r_{2}+r_{3} \leq C_{b}=C_{b}\left(x_{b}, z_{b}\right),
$$

which fits in the format of (1). Under this model, one can then attempt to jointly attempt to optimize the transmit powers $x_{a}$ and $x_{b}$ along with the flow rates $r_{1}, r_{2}$ and $r_{3}$ to maximize some utility.

To summarize the optimization, let $\boldsymbol{\theta}$ denote the vector of all the decisions variables

$$
\boldsymbol{\theta}=(\mathbf{r}, \mathbf{x}, \mathbf{z}) .
$$

and let $\overline{\boldsymbol{\theta}}$ denote the maximum values

$$
\overline{\boldsymbol{\theta}}=(\overline{\mathbf{r}}, \overline{\mathbf{x}}, \overline{\mathbf{z}}) .
$$

With some abuse of notation, we let $U(\boldsymbol{\theta})=U(\mathbf{r})$, so that we can rewrite the optimization as

$$
\max _{0 \leq \boldsymbol{\theta} \leq \overline{\boldsymbol{\theta}}} U(\boldsymbol{\theta}) \quad \text { s.t. } g(\boldsymbol{\theta}) \leq 0,
$$

where $g(\boldsymbol{\theta})$ is the vector of constraints

$$
g(\boldsymbol{\theta}):=\left[\begin{array}{c}
\mathbf{A}_{x} \mathbf{x}-\mathbf{b}_{x} \\
\mathbf{A}_{r} \mathbf{r}-\mathbf{b}_{r} \\
\mathbf{G} \mathbf{x}-\mathbf{z} \\
\mathbf{E} \mathbf{r}-C(\mathbf{x}, \mathbf{z})
\end{array}\right],
$$

and $\overline{\boldsymbol{\theta}}$ is some upper bound on the variables.

\section{User Association AND Multiflow in CELlular NETWORKS}

Having described the general flow and interference control problem, we now turn to special case of user association and bandwidth optimization in cellular systems. Our model considers the downlink of a cellular network with $N_{\text {MS }}$ mobile stations, MS $i, i=1, \ldots, N_{\mathrm{MS}}$ and $N_{\mathrm{BS}}$ base stations, BS $j$, $j=1, \ldots, N_{\mathrm{BS}}$. We suppose that there are a total of $L$ flows, with each flow $\ell$ passing through exactly one BS-MS pair. Although a single base station may support multiple flows, we will logically regard each flow $\ell$ as a separate wireless link with rate $r_{\ell}$ and bandwidth allocation $x_{\ell}$. For simplicity, we will assume only the BS-MS links are wireless with interference constraints. Any other (wired) links are assumed to have fixed capacity constraints. We let $\Gamma_{\mathrm{MS}}(i)$ denote the set of flows received by MS $i$ and $\Gamma_{\mathrm{BS}}(j)$ be the set of flows transmitted by $\mathrm{BS} j$.

To illustrate the model and notation, Fig. 2 shows a simple example of a network with two mobile stations, MS1 and MS2, 


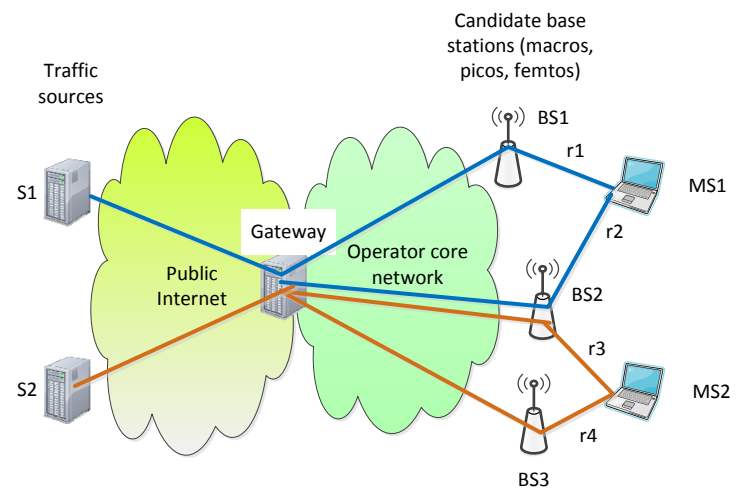

Fig. 2: Simple example network where two mobiles, MS1 and MS2, must each select between two base stations. Since each of the two MS's have two candidate flows, there are a total of $L=4$ flows with sets $\Gamma_{M S}(1)=\{1,2\}, \Gamma_{M S}(2)=\{3,4\}$, $\Gamma_{B S}(1)=\{1\}, \Gamma_{B S}(2)=\{2,3\}$ and $\Gamma_{B S}(3)=\{4\}$.

in a typical cellular configuration. Mobiles MS1 and MS2 received data from sources $\mathrm{S} 1$ and $\mathrm{S} 2$ in the public Internet. Following a standard cellular architecture such as 3GPP LTE [31], the data is assumed to arrive from the public Internet to a common gateway which then tunnels the traffic through an operator-controlled core network to the base station selected to the serve the mobiles. In the simple example in Fig. 2 we have three candidate base stations shared amongst the two mobiles. Since each of the two MS's have two candidate BSs, there are a total of $L=4$ flows, each flow following a path of the form: source $\rightarrow$ gateway $\rightarrow \mathrm{BS} \rightarrow \mathrm{MS}$.

Now returning to the general case, let $\bar{r}_{i}$ be the total rate to MS $i$, which must satisfy the constraint

$$
\bar{r}_{i} \leq \sum_{\ell \in \Gamma_{\mathrm{MS}}(i)} r_{\ell}, \quad i=1, \ldots, N_{\mathrm{MS}}
$$

We let $\mathbf{r}$ be the vector containing both the total and individual flow rates

$$
\mathbf{r}=\left(\bar{r}_{1}, \ldots, \bar{r}_{N_{\mathrm{MS}}}, r_{1}, \ldots, r_{L}\right)^{T} .
$$

We assume the utility is a separable function of the total rates to each MS so that

$$
U(\mathbf{r})=\sum_{i=1}^{N_{\mathrm{MS}}} U_{i}\left(\bar{r}_{i}\right)
$$

for some utility functions $U_{i}\left(\bar{r}_{i}\right)$.

To model the bandwidth constraints and interference, we assume that each BS $j$ has a total available bandwidth $\bar{w}_{j}$ and radiates a fixed power per unit bandwidth $P_{j}$. We let $x_{\ell}$ be the bandwidth allocated to the flow on link $\ell$. The bandwidth allocations at BS $j$ must thus satisfy the constraint

$$
\sum_{\ell \in \Gamma_{\mathrm{BS}}(j)} x_{\ell} \leq \bar{w}_{j}, \quad j=1, \ldots, N_{\mathrm{BS}} .
$$

Also, the total power radiated by $\mathrm{BS} j$ is given by

$$
P_{j} \sum_{\ell \in \Gamma_{\mathrm{BS}}(j)} x_{\ell}
$$

Now suppose that a flow $\ell$ is served by BS $j$ and received by MS $i$. Then, the total interference power received on that flow is given by

$$
z_{\ell}=\sum_{k \neq j} H_{\ell k} P_{k} \sum_{\ell \in \Gamma_{\mathrm{BS}}(k)} x_{\ell}
$$

where $H_{\ell k}$ is the channel gain from BSk to the receiver for flow $\ell$. Since flow $\ell$ is served by $\mathrm{BS} j$, the SINR on the link is then

$$
\gamma_{\ell}\left(z_{\ell}\right)=\frac{H_{\ell j} P_{j}}{z_{\ell}+N_{\ell}}
$$

where $N_{\ell}$ is the thermal noise at the receiver. We can then assume the capacity constraint on the $\ell$-th link is of the form

$$
r_{\ell} \leq C\left(x_{\ell}, z_{\ell}\right):=x_{\ell} \rho_{\ell}\left(\gamma_{\ell}\left(z_{\ell}\right)\right)
$$

where $\rho_{\ell}$ is the spectral efficiency (rate per unit bandwidth) on the $\ell$-th link as a function of the SINR. The spectral efficiency $\rho_{\ell}$ is multiplied by the allocated bandwidth $x_{\ell}$ to yield the link capacity $C_{\ell}\left(x_{\ell}, z_{\ell}\right)$ in 16. Our formulation will permit any continuous spectral efficiency function.

Under this model, we consider two problems:

- Multiflow optimization: In this case, there are no additional restrictions on the rate vector $r$ so that the mobile can be served from multiple base station cells simultaneously. In 3GPP LTE-Advanced, this scenario would correspond to carrier aggregation [10], [11].

- User association optimization: In this case, we assume each mobile can be served by only one cell at a time, which is the standard model for LTE without the advanced carrier aggregation feature. This requirement can be modeled via an additional constraint: for all $i=1, \ldots, N_{\mathrm{MS}}$,

$$
r_{\ell}=0 \text { for all but one } \ell \in \Gamma_{\mathrm{MS}}(i) \text {. }
$$

Under this constraint, the selection of the index $\ell$ such that $r_{\ell} \neq 0$, determines which BS will serve MS $i$.

Now, the multiflow optimization is exactly a special case of the general flow and interference control problem in Section II Specifically, let $\mathbf{x} \in \mathbb{R}^{L}$ be the vector of bandwidth allocations and $\mathbf{z} \in \mathbb{R}^{L}$ be the vector of interference powers. The bandwidth constraints (14) can be written of the form $\mathbf{A}_{x} \mathbf{x} \leq \mathbf{b}_{x}$ for some $\mathbf{A}_{x}$ and $\mathbf{b}_{x}$. The network constraints will be written in the form $\mathbf{A}_{r} \mathbf{r} \leq \mathbf{b}_{r}$, which can include constraints on either the flow rates or total rates. The interference powers (15) can be written as $\mathbf{z}=\mathbf{G} \mathbf{x}$ for some gain matrix $\mathbf{G}$ and the capacity (16) can be written in the form (4).

On the other hand, the single path constraint (17) for the user association optimization problem cannot be directly placed into the form of flow and interference control problem in Section III As we will discuss in the next section, we will use the multiflow optimization as an upper bound for this problem and then truncate the output to obtain a feasible single path solution. 


\section{APPROXIMATE OPTIMIZATION VIA DUAL DECOMPOSITION}

\section{A. General Flow and Interference Control}

We now describe potential methods for solving the optimization problems. We begin with the general flow and interference control problem in Section II The maximization of (9) in this problem is non-convex in general since the capacity function $C(\mathbf{x}, \mathbf{z})$ may have an arbitrary form. In addition, the utility function $U(\mathbf{r})$ may be non-concave. However, since both the objective functions and constraints in (9) admit a separable structure, it is natural to attempt to solve the (9) via dual decomposition.

To this end, corresponding to the constrained optimization (9), define the Lagrangian [32]

$$
\begin{aligned}
& L(\boldsymbol{\theta}, \boldsymbol{\mu}):=U(\boldsymbol{\theta})-\boldsymbol{\mu}^{T} g(\boldsymbol{\theta}) \\
& \quad=U(\mathbf{r})+\boldsymbol{\mu}^{x T}\left(\mathbf{b}_{x}-\mathbf{A}_{x} \mathbf{x}\right)+\boldsymbol{\mu}^{r T}\left(\mathbf{b}_{r}-\mathbf{A}_{r} \mathbf{r}\right) \\
& \quad+\boldsymbol{\mu}^{z T}(\mathbf{z}-\mathbf{G x})+\boldsymbol{\mu}^{c T}(C(\mathbf{x}, \mathbf{z})-\mathbf{E r}),
\end{aligned}
$$

where $\boldsymbol{\mu} \geq 0$ are the dual parameters which are partitioned conformably with $g(\boldsymbol{\theta})$ in $(10)$ as

$$
\boldsymbol{\mu}=\left(\boldsymbol{\mu}^{x}, \boldsymbol{\mu}^{r}, \boldsymbol{\mu}^{z}, \boldsymbol{\mu}^{c}\right)^{T} .
$$

The following simple lemma provides our main motivation for considering duality-based optimizations: namely that the dual maximization is separable:

Lemma 1: Let $\Phi(\boldsymbol{\theta})$ be any separable function of the form

$$
\Phi(\boldsymbol{\theta})=\Phi(\mathbf{r}, \mathbf{x}, \mathbf{z}):=\sum_{i} \phi_{i}^{r}\left(r_{i}\right)+\sum_{\ell} \phi_{\ell}^{x}\left(x_{\ell}, z_{\ell}\right),
$$

for some functions $\phi_{i}^{r}(\cdot)$ and $\phi_{\ell}^{x}(\cdot)$. Then, the maximization of the Lagrangian $L(\boldsymbol{\theta}, \boldsymbol{\mu})$ in (18) augmented by $\Phi(\boldsymbol{\theta})$,

$$
\widehat{\boldsymbol{\theta}}(\boldsymbol{\mu})=(\widehat{\mathbf{r}}(\boldsymbol{\mu}), \widehat{\mathbf{x}}(\boldsymbol{\mu}), \widehat{\mathbf{z}}(\boldsymbol{\mu})):=\underset{\boldsymbol{\theta} \leq \overline{\boldsymbol{\theta}}}{\arg \max }[L(\boldsymbol{\theta}, \boldsymbol{\mu})-\Phi(\boldsymbol{\theta})]
$$

has components given by the solutions to the optimizations

$$
\begin{gathered}
\widehat{r}_{s} \in \underset{r_{s}}{\arg \max }\left[U_{s}\left(r_{s}\right)-\phi_{s}^{r}\left(r_{s}\right)-\left(\mathbf{E}^{T} \boldsymbol{\mu}^{c}+\mathbf{A}_{r}^{T} \boldsymbol{\mu}^{r}\right)_{s} r_{s}\right] \\
\text { s.t. } \quad 0 \leq r_{s} \leq \bar{r}_{s}
\end{gathered}
$$

and

$$
\begin{aligned}
\left(\widehat{x}_{\ell}, \widehat{z}_{\ell}\right) \in \underset{x_{\ell}, z_{\ell}}{\arg \max }\left[\mu_{\ell}^{c} C_{\ell}\left(x_{\ell}, z_{\ell}\right)-\phi_{\ell}^{x}\left(x_{\ell}\right)-\phi_{\ell}^{z}\left(z_{\ell}\right)\right. \\
\left.-\left(\mathbf{A}_{x}^{T} \boldsymbol{\mu}^{x}+\mathbf{G}^{T} \boldsymbol{\mu}^{z}\right)_{\ell} x_{\ell}+\mu_{\ell}^{z} z_{\ell}\right] \\
\text { s.t. } 0 \leq x_{\ell} \leq \bar{x}_{\ell}, \quad 0 \leq z_{\ell} \leq \bar{z}_{\ell} .
\end{aligned}
$$

Proof: This result follows immediately from the separable structure of the objective function and constraints.

Thus, the vector-valued optimization (21) decomposes into $S$ one-dimensional optimizations over the variables $r_{s}$ and $L$ two-dimensional optimizations over the variables $\left(x_{\ell}, z_{\ell}\right)$. In many cases, we will see that these have explicit closed-form solutions or can be easily computed via a simple line search.

There are two key consequences to this separability result: a) Computation of an Upper Bound: First, the wellknown weak duality property [32] states that an upper bound to the maximum of the utility is given by

$$
\max _{\boldsymbol{\theta}: g(\boldsymbol{\theta}) \leq 0} U(\boldsymbol{\theta}) \leq \min _{\boldsymbol{\mu} \geq 0} \bar{L}(\boldsymbol{\mu})
$$

where $\bar{L}(\boldsymbol{\mu})$ is the dual maxima,

$$
\bar{L}(\boldsymbol{\mu}):=\max _{\boldsymbol{\theta} \leq \overline{\boldsymbol{\theta}}} L(\boldsymbol{\theta}, \boldsymbol{\mu}) .
$$

This bound applies even when $U(\boldsymbol{\theta})$ is non-convex. Moreover, $\bar{L}(\boldsymbol{\mu})$ is also always convex in $\boldsymbol{\mu}$ with subgradient $g(\boldsymbol{\theta}(\boldsymbol{\mu}))$. Since Lemma 1 shows that the maxima $\widehat{\boldsymbol{\theta}}(\boldsymbol{\mu})$ and $\bar{L}(\boldsymbol{\mu})$ can be easily evaluated for each $\mu$, the upper bound in (24) can be minimized via convex programming, thereby providing a computationally tractable upper bound on the utility. Of course, since $U(\boldsymbol{\theta})$ and/or the constraints may be non-convex, the bound may not be tight (i.e. the problem is not strongly dual). Also, in absence of the augmenting term, the subgradient may be non-smooth, meaning the convex optimization may have a slower (although still feasible) convergence rate [33], [34].

b) Augmented Lagrangian Optimization: Secondly, since the maximization 21 can be computed for any $\boldsymbol{\mu}$ and separable augmenting function $\Phi$, one can employ a wide range of augmented Lagrangian optimization methods for finding local optima for the constrained maxima problem (9). Augmented Lagrangian methods [32] generate a sequence of estimates $\boldsymbol{\theta}^{t}$ typically through the iterations of the form

$$
\begin{aligned}
\boldsymbol{\theta}^{t+1} & =\underset{\boldsymbol{\theta} \leq \overline{\boldsymbol{\theta}}}{\arg \max }\left[L\left(\boldsymbol{\theta}, \boldsymbol{\mu}^{t}\right)-\Phi\left(\boldsymbol{\theta}, \boldsymbol{\theta}^{t}\right)\right] \\
\boldsymbol{\mu}^{t+1} & =\boldsymbol{\mu}^{t}-\alpha g\left(\boldsymbol{\theta}^{t+1}\right),
\end{aligned}
$$

where $\alpha>0$ is a step-size and $\Phi\left(\boldsymbol{\theta}, \boldsymbol{\theta}^{t}\right) \geq 0$ is a so-called augmenting function that smooths the optimization. Separable forms of the augmenting function can include the quadratic $\Phi\left(\boldsymbol{\theta}, \boldsymbol{\theta}^{t}\right)=\epsilon\left\|\boldsymbol{\theta}-\boldsymbol{\theta}^{t}\right\|_{2}^{2}$ for some $\epsilon>0$.

\section{B. Multiflow Optimization}

Since the multiflow cellular optimization in Section III is a special case of the general flow and interference control problem, we can directly apply the results above in Section IV-A to compute upper bounds on the utility as well as efficiently implement augmented Lagrangian techniques.

In addition, in the cellular context augmented Lagrangian iterations of the form (26) also admit a simple distributed implementation - a feature also found in the similar user association optimization algorithm in [4], as well as many duality-based power control algorithms (see the monograph [30]). Specifically, it can be verified that, using the separable structure of the optimization in Lemma 1, the rates $r_{\ell}$ and bandwidth allocations $x_{\ell}$ and target interference level $z_{\ell}$ for each link can be computed at the base station serving that flow. The base station only needs local information on the gain matrix $\mathbf{G}$ and dual parameters. The base stations can then update the dual parameters $\boldsymbol{\mu}$ as in (26b) and broadcast the values to the neighboring base stations potentially through the mobiles. 
TABLE I: Simulation parameters

\begin{tabular}{|l|l|}
\hline Parameter & \multicolumn{1}{|c|}{ Value } \\
\hline \hline Macro layout & $\begin{array}{l}19 \text { hexagonal cell sites with wraparound, 3 cells } \\
\text { per site. Inter-site distance=500 m. }\end{array}$ \\
\hline $\begin{array}{l}\text { Pico and UE distri- } \\
\text { bution }\end{array}$ & $\begin{array}{l}10 \text { picos and 10 or 25 UEs per macrocell, uni- } \\
\text { formly distributed. }\end{array}$ \\
\hline TX power & $46 \mathrm{dBm}$ (macro), 30 dBm (pico) \\
\hline Path loss & $\begin{array}{l}\text { macro: } 131.1+42.8 \log _{10}(R), \\
\text { pico: } 145.4+37.5 \log _{10}(R)\end{array}$ \\
\hline Carrier frequency & $2.1 \mathrm{GHz}$ \\
\hline Shadowing & $8 \mathrm{~dB}$ lognormal std. dev. \\
\hline UE noise figure & $7 \mathrm{~dB}$ \\
\hline System bandwidth & $10 \mathrm{MHz}$ \\
\hline SNR to rate & $3 \mathrm{~dB}$ from Shannon, max 4.8 bps/Hz $\mid 35$ \\
\hline Antenna Pattern & $\begin{array}{l}\text { macro: 3GPP standard model } \\
\text { pico: omnidirectional }\end{array}$ \\
\hline Traffic model & Full buffer \\
\hline
\end{tabular}

\section{User Association Optimization}

As discussed above, the single path constraint (17) is nonlinear and cannot be placed into the framework of the general flow and interference control problem in Section II. However, a standard method [12]-[14] is to solve the multiflow upper bound and then simply the "truncate" the solution to remove all but the best path. In most cases, the multiflow optimization yields solutions that are zero in all but one path (the book [14] provides conditions under which this property is provably true) and therefore the truncation often does not significantly alter the optimization result. A similar truncation method is used in [4].

\section{Numerical Simulations}

\section{A. Lightly Loading Heterogeneous Network}

To demonstrate the methodology we simulated the algorithm in a two-tier heterogeneous network with macro- and picocells loosely following the 3GPP evaluation methodology in [23], specifically the macro and outdoor remote radio head/hotzone scenario in Configuration \#1. The parameters are shown in Table I. The main difference is that we consider a much greater density of picocells (10 instead of 4 picos per macrocell), making the number of UEs per cell small. We will see that under this lightly-loaded scenario, the power optimization can offer significant benefits.

We focus on the problem of optimized user association (as opposed to multiflow) where each UE must select exactly one cell - either macro or pico - since this is main concern in the cellular standards. To apply the proposed optimization method to the user association problem, after the drop, each UE selected three candidate BSs: the strongest macrocell and the two strongest picocells. The algorithm was then run to maximize a proportional fair utility, $U_{i}(r)=\log (r)$, which corresponds to the sum-log rate. Fig. 3 compares the resulting rate distribution from the optimization algorithm against simpler, but standard, cell selection policies. The curve labeled "optimizer" is the optimization run, but keeping all the cells transmitting at maximum power. In this case, the spectral efficiency is constant and the algorithm reduces to

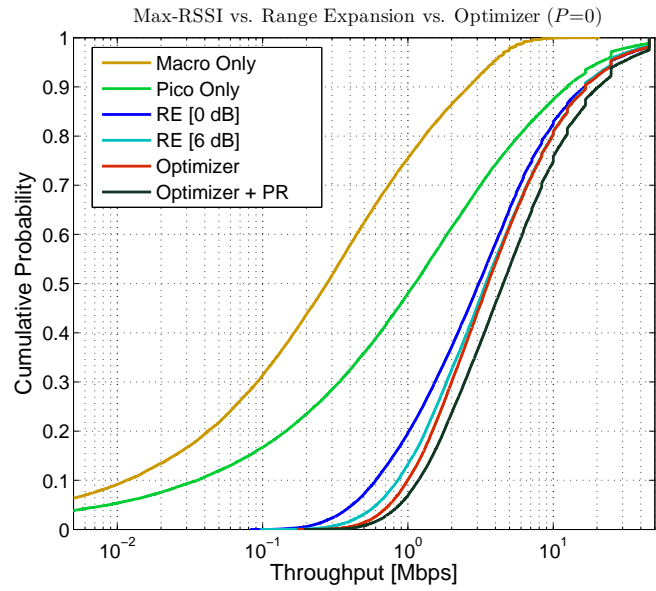

Fig. 3: Rate distribution comparing various user association algorithms for $10 \mathrm{UEs}$ per macrocell. Other parameters given in Table I

the method of [4]. The curve labeled "optimizer+PR" adds power reduction where reducing the bandwidth allocated in a cell causes its power to reduce. For comparison, the figure also plots the "macro only" and "pico only" where the UEs use only the macro- or picocells. Both of these options give very poor rates. The range expansion methods (labeled RE) select the strongest cell - pico or macro - but the picocells are given a fixed bias in the received signal strength to encourage mobiles to select the smaller cell. Manual trials with different bias levels found an an optimal bias of approximately $6 \mathrm{~dB}$. Fig. 3 shows the rate distribution of RE with bias of 0 and 6 dB.

Table III shows the numerical values from the simulation. With the light loading of 10 UEs per macrocell (the case plotted in Fig. 3, we see that the proposed optimization with power reduction offers an approximately $23 \%$ increase in cell throughput and $42 \%$ increase in the $5 \%$ cell edge capacity. While not dramatic, the gains are larger than previous studies [3], [4] which showed little gain over simple RE with the correct bias. The reason for the larger gain here is that, since we are considering a low UE to cell ratio, some picocells can be chosen to be unused and can reduce their power. Indeed, if we go to a higher loading of 25 UEs per macrocell (2.5 UEs per picocell), the gains from power reduction are reduced since most of the picocell become fully utilized.

\section{B. Backhaul Network Constraints}

In addition to the interference control, a second appealing feature of the proposed user association methodology is that it can account for backhaul network constraints. Such backhaul constraints may arise when open femtocells are used for coverage. Femtocells are not connected directly into the operator core network, but instead use third party backhaul which may be variable in quality. To simulate this scenario, we employed the same assumptions as before, but we placed a 1 Mbps limit on $50 \%$ of the picocell links (the picocells now modeling large numbers of open femtocells). Fig. 4 shows that the gains from optimized user association in this scenario are even larger. 
TABLE II: Simulation Results

\begin{tabular}{|c|c|c|l|l|}
\hline \multicolumn{2}{|c|}{ Method } & RE [6dB] & \multicolumn{1}{c|}{ Opt. } & \multicolumn{1}{c|}{ Opt. + PR } \\
\hline \multirow{2}{*}{ 10UE } & Sp. Eff. $^{1}$ & 0.608 & $0.625(2.8 \%)^{3}$ & $0.748(23 \%)^{4}$ \\
\cline { 2 - 5 } & 5\% Rate & & \\
& 2 & 0.608 & $0.745(22.6 \%)$ & $0.866(42.4 \%)$ \\
\hline \multirow{2}{*}{ 25UE } & Sp. Eff. & 0.909 & $0.940(3.4 \%)$ & $0.989(8.7 \%)$ \\
\cline { 2 - 5 } & 5\% Rate & 0.351 & $0.418(19.1 \%)$ & $0.435(23.9 \%)$ \\
\hline
\end{tabular}

$1:$ Spectral efficiency in bit $/ \mathrm{sec} / \mathrm{Hz}$

$2: 5 \%$ Cell-edge rate in Mbps

3,4 : gains compared to range expansion $[6 \mathrm{~dB}]$

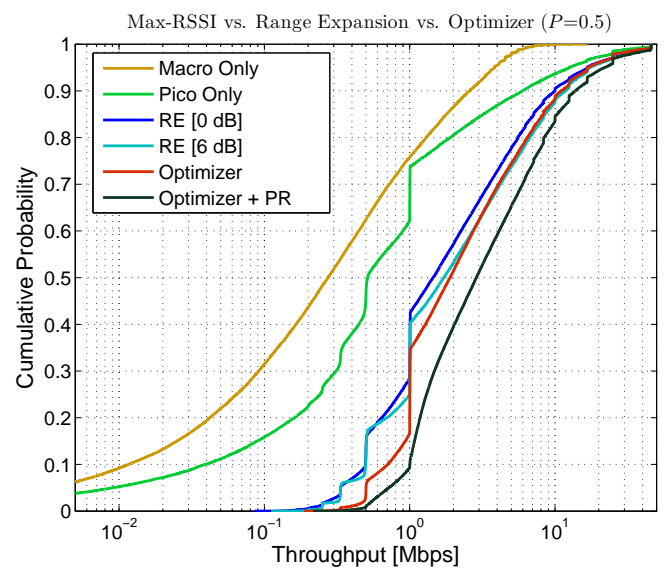

Fig. 4: Rate distribution of various user association algorithms with backhaul rate limits on $50 \%$ of the picocells.

Indeed, for 10 UEs per cell, there is an $28 \%$ increase in average user throughput and $112 \%$ increase in the 5\% cell-edge rate. In addition, Fig. 4 shows that RE gives a minimal gains in this heterogeneous network condition since RE is a powerbased selection scheme and does not account for the backhaul constraints.

\section{CONCLUSION}

We have presented a general methodology for joint interference coordination and user association problems in cellular networks. The methodology is extremely general in that it can incorporate interference constraints described by an linear mixing model followed by an arbitrary interference-torate mapping. Although we have only considered bandwidth allocations in this paper, based on the results in [15], we believe that we can extend these results to more complex ICIC schemes as well such as subband allocations and beamforming. Also, while we cannot find a provably optimal solution, the dual decomposition method enables efficient implementation of suboptimal solutions via augmented Lagrangian techniques as well as computation of upper bounds on the maximum utility.

\section{REFERENCES}

[1] Qualcomm, "LTE Advanced: Heterogeneous networks," Whitepaper available online at http://www.qualcomm.com/media/documents, Jan. 2011.

[2] A. Damnjanovic, J. Montojo, Y. Wei, T. Ji, T. Luo, M. Vajapeyam, T. Yoo, O. Song, and D. Malladi, "A survey on 3GPP heterogeneous networks," IEEE Wireless Communications, vol. 18, no. 3, pp. 10-21, 2011.

[3] I. Guvenc, M.-R. Jeong, I. Demirdogen, B. Kecicioglu, and F. Watanabe, "Range expansion and Inter-Cell Interference Coordination (ICIC) for picocell networks," Proc. IEEE Veh. Tech. Conf., pp. 1-6.
[4] Q. Ye, B. Rong, Y. Chen, M. Al-Shalash, C. Caramanis, and J. G. Andrews, "User association for load balancing in heterogeneous cellular networks," IEEE Trans. Wireless Comm., Dec. 2013.

[5] V. Chandrasekhar, J. G. Andrews, and A. Gatherer, "Femtocell networks: A survey," IEEE Comm. Mag., vol. 46, no. 9, pp. 59-67, Sep. 2009.

[6] J. G. Andrews, H. Claussen, M. Dohler, S. Rangan, and M. C. Reed, "Femtocells: Past, present, and future," IEEE J. Sel. Areas Comm., vol. 30, no. 3, Apr. 2012.

[7] 3GPP, "New Work Item Proposal: Enhanced ICIC for non-CA based deployments of heterogeneous networks for LTE," RP-100372, 2010.

[8] F. P. Kelly, A. K. Maulloo, and D. K. H. Tan, "Rate control for communication networks: shadow prices, proportional fairness and stability," Journal of the Operational Research Society, vol. 49, no. 3, pp. 237-252, Mar. 1998.

[9] S. Shakkottai and R. Srikant, Network Optimization and Control, ser Foundations and Trends in Networking. NOW Publishers, 2007.

[10] 3GPP, "Evolved Universal Terrestrial Radio Access (E-UTRA) and Evolved Universal Terrestrial Radio Access Network (E-UTRAN); Overall description; Stage 2," TS 36.300 (release 10), 2010.

[11] G. Yuan, X. Zhang, W. Wang, and Y. Yang, "Carrier aggregation for LTE-Advanced mobile communication systems," IEEE Comm. Mag., vol. 48, no. 2, pp. 88-93, 2010.

[12] L. Ford and D. R. Fullkerson, Flows in Networking. Princeton University Press, 1958.

[13] R. E. Gomroy and T. C. Hu, "Multiterminal network flows," SIAM J. Applied Math, vol. 9, pp. 551-570, 1961.

[14] M. Pióro and D. Medhi, Routing, Flow and Capacity Design in Communication and Computer Networks. Elsevier, 2004.

[15] S. Rangan and R. Madan, "Belief propagation methods for intercell interference coordination in femtocell networks," IEEE J. Sel. Areas Comm., vol. 30, no. 3, pp. 631-640, Apr. 2012.

[16] S. Hanly, "An algorithm for combined cell-site selection and power control to maximize cellular spread spectrum capacity," IEEE J. Sel. Areas Comm., vol. 13, no. 7, pp. 2796-2815, September 1995.

[17] L. Smolyar, I. Bergel, and H. Messer, "Unified approach to joint power allocation and base assignment in nonorthogonal networks," IEEE Trans. on Vehicular Technology, vol. 58, no. 8, Oct. 2009.

[18] R. Madan, J. Borran, A. Sampath, N. Bhushan, A. Khandekar, and T. Ji, "Cell association and interference coordination in heterogeneous LTE-A cellular networks," IEEE J. Sel. Areas Comm., vol. 28, no. 9, Dec. 2010.

[19] Qualcomm Europe, "Range expansion for efficient support of heterogeneous networks," 3GPP TSG-RAN WG1 54, R1-083813, Sep. 2008.

[20] - "DL performance with hotzone cells," 3GPP TSG-RAN WG1 58, R1-094225, Oct. 2009.

[21] H. Galeana, A. Lainz, and R. Ferrus, "User allocation algorithm with rate guarantees for multi-rate mobile networks with backhaul constraints," Proc. IEEE Veh. Tech. Conf., pp. 1-5, 2009.

[22] H. Kim, G. de Veciana, X. Yang, and M. Venkatachalam, " $\alpha$-optimal user association and cell load balancing in wireless networks," Proc. INFOCOMM, pp. 1-5, 2010.

[23] 3GPP, "Evolved Universal Terrestrial Radio Access (E-UTRA); Further advancements for E-UTRA physical layer aspects," TR 36.814 (release 9), 2010.

[24] H.-S. Jo, Y. J. Sang, P. Xia, and J. Andrews, "Heterogeneous cellular networks with flexible cell association: A comprehensive downlink SINR analysis," IEEE Trans. Wireless Comm, vol. 11, no. 10, pp. 3484-3495, 2012.

[25] J. K. MacKie-mason and H. R. Varian, "Pricing congestible network resources," IEEE J. Sel. Areas Comm., vol. 13, no. 7, pp. 1141 - 1149, Sep. 1995.

[26] K. Kar, S. Sarkar, and L. Tassiulas, "Optimization based rate control for multipath sessions," Inst. Syst. Research, Tech. Rep. 2001-1, 2001.

[27] X. Lin and N. B. Shroff, "Utility maximization for communication networks with multipath routing," IEEE Trans. Automat. Control, vol. 51, no. 5, pp. 766 - 781, May 2006.

[28] M. Chiang, "Balancing transport and physical layers in wireless multihop networks: Jointly optimal congestion control and power control," IEEE J. Sel. Areas Comm., vol. 23, no. 1, pp. 104-116, January 2005.

[29] P. Hande, S. Rangan, M. Chiang, and X. Wu, "Distributed uplink power control for optimal SIR assignment in cellular data networks," IEEE/ACM Trans. Networking, vol. 16, no. 6, pp. 1420-1433, Dec. 2008

[30] M. Chiang, P. Hande, T. Lan, and C. W. Tan, "Power control in wireless cellular networks," Foundation and Trends in Networking, vol. 2, no. 4, pp. 381-533, Jul. 2008.

[31] M. Olsson, S. Sultana, S. Rommer, L. Frid, and C. Mulligan, SAE and the Evolved Packet Core: Driving the Mobile Broadband Revolution. Academic Press, 2009. 
[32] J. Nocedal and S. J. Wright, Numerical Optimization. Springer Verlag, 2006.

[33] N. Shor, Minimization Methods for Non-Differentiable Functions. Springer Verlag, 1985.

[34] Y. Nesterov, "Smooth minimization of non-smooth functions," Mathematical Programming, vol. 103, no. 1, p. 127152, 2005.

[35] P. Mogensen, W. Na, I. Z. Kovács, F. Frederiksen, A. Pokhariyal, K. I. Pedersen, T. Kolding, K. Hugl, and M. Kuusela, "LTE capacity compared to the Shannon bound," in Proc. IEEE Veh. Tech. Conf., 2007. 